[Preprint version, please cite as] Alessio Ishizaka, Nam Nguyen Hoang, Calibrated Fuzzy AHP for current bank account selection, Expert Systems with Applications, DOI 10.1016/j.eswa.2012.12.089, advance online publication

\title{
Calibrated Fuzzy AHP for current bank account selection
}

\author{
Alessio Ishizaka ${ }^{1}$, Nam Nguyen Hoang \\ University of Portsmouth, Portsmouth Business School, Richmond Building, Portland Street, \\ Portsmouth PO1 3DE, United Kingdom \\ Alessio.Ishizaka@port.ac.uk, nhnam1988@gmail.com
}

\begin{abstract}
:
Fuzzy AHP is a hybrid method that combines Fuzzy Set Theory and AHP. It has been developed to take into account uncertainty and imprecision in the evaluations. Fuzzy Set Theory requires the definition of a membership function. At present, there are no indications of how these membership functions can be constructed. In this paper, a way to calibrate the membership functions with comparisons given by the decision-maker on alternatives with known measures is proposed. This new technique is illustrated in a study measuring the most important factors in selecting a student current account.
\end{abstract}

Keywords: Fuzzy AHP, membership functions, customisation, current account, banking

\section{Introduction}

Despite the popularity and simplicity of the Analytic Hierarchy Process (AHP), it is often criticised for its inability to adequately handle the uncertainty of a decision maker's preferences. In classic AHP, the judgements are represented by exact values on a scale of 1 to 9 (Saaty, 1977, 1980). However, in many real cases, the linguistic assessments of human evaluations are often vague, and it is not realistic to represent them with crisps values. To overcome these shortcomings, fuzzy AHP has been developed to take into account this uncertainty and imprecision. It is essentially the combination of two methods: fuzzy set theory and AHP (Van Laarhoven \& Pedrycz, 1983). Fuzzy set theory requires the definition of a membership function for each verbal judgement. However, in all papers reviewed, there was no indication of how the membership functions have been selected. This paper proposes a way to calibrate the membership functions with comparisons given by the decision-maker on alternatives with known measures. In this case, we asked to compare the surface of geometrical figures and as a result, the membership function was personalised for each participant. Then, the fuzzy AHP with the customised membership functions is applied to a case study in order to establish the most important factors in the selection of a student current account. We found that service is the most weighted criteria when selecting a bank account.

\footnotetext{
${ }^{1}$ Corresponding author : 00442392844171
} 
[Preprint version, please cite as] Alessio Ishizaka, Nam Nguyen Hoang, Calibrated Fuzzy AHP for current bank account selection, Expert Systems with Applications, DOI 10.1016/j.eswa.2012.12.089, advance online publication

\section{Fuzzy AHP}

Fuzzy AHP was first proposed by Van Laarhoven and Pedrycz (1983) and is an extension of AHP combined with fuzzy set theory (Zadeh, 1965). The main advantage of this combination is that it makes allowances for the vagueness and imprecision of human preference. The key idea is that a certain degree of an element belongs to a fuzzy membership set, which is given by a function depicted on a two-axis diagram. The horizontal axis consists of the domain elements of the fuzzy sets and the vertical axis the degree of membership on a scale of 0 to 1 . These membership functions can take several shapes: linear, S-curves, triangular or trapezoidal representations. In practice, triangular and trapezoidal membership functions are the most frequently used. They can be denoted by $\tilde{\mathrm{A}}=\left(l, m_{l}, m_{u}, u\right)$, where $l \leq$ $m_{l} \leq m_{u} \leq u$ correspond to lower, modal-lower, modal-upper and upper bound, i.e. the trapezium's angle points. If the membership is triangular, then $m_{l}=m_{u}$ (Figure 1). The membership of $\tilde{\mathrm{A}}$ is defined by:

$\mu_{\tilde{A}}(x)=\left\{\begin{array}{cc}\frac{x-l}{m-l}, & l \leq x \leq m \\ 1, & x=m \\ \frac{u-x}{u-m}, & m<x<u \\ 0, & \text { otherwise }\end{array}\right.$

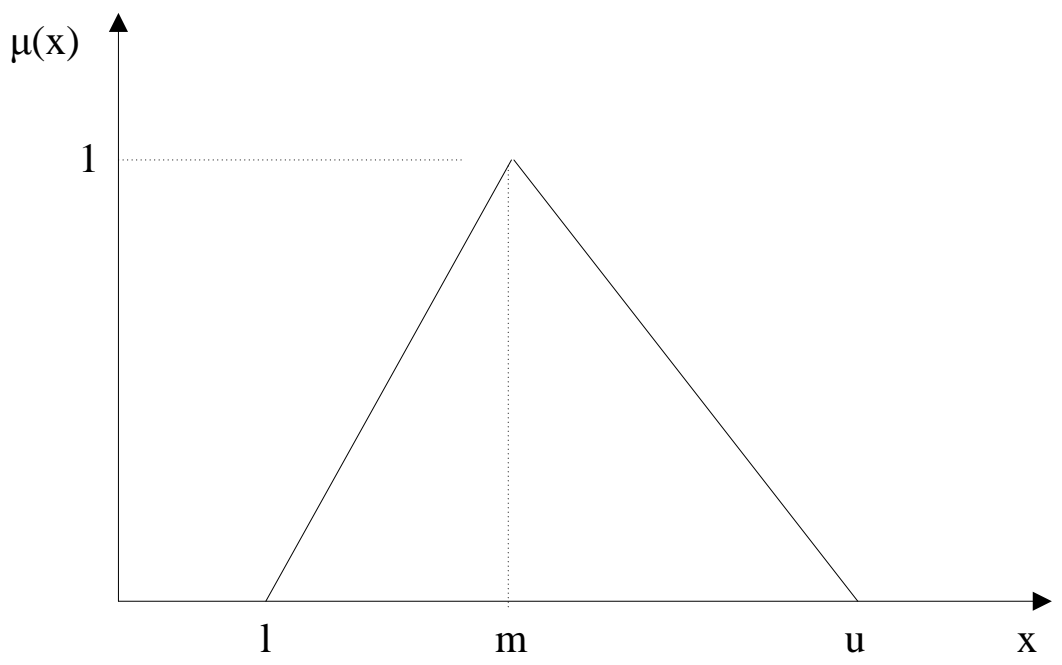

Figure 1: Trapezoidal membership function

Fuzzy AHP is based on 4 steps:

a) For each linguistic term of the evaluation scale, a membership function is constructed.

b) Criteria/alternatives are pair-wise compared in comparison matrix $\tilde{\mathrm{A}}$. 
[Preprint version, please cite as] Alessio Ishizaka, Nam Nguyen Hoang, Calibrated Fuzzy AHP for current bank account selection, Expert Systems with Applications, DOI 10.1016/j.eswa.2012.12.089, advance online publication

$$
\tilde{\mathrm{A}}=\left[\begin{array}{cccc}
\tilde{a}_{11} & \tilde{a}_{12} & \ldots & \tilde{a}_{1 n} \\
\tilde{a}_{21} & \tilde{a}_{22} & \ldots & \tilde{a}_{2 n} \\
\ldots & \ldots & \ldots & \ldots \\
\tilde{a}_{n 1} & \tilde{a}_{n 2} & \ldots & \tilde{a}_{n n}
\end{array}\right]
$$

where $\tilde{a}_{i j}$ is the fuzzy comparison between criterion/alternative $i$ and $j$

c) Fuzzy priorities are derived from comparison matrix Ã. This is done using the eigenvalue method (2) or any other method used in traditional AHP (Ishizaka \& Labib, 2011).

$$
\tilde{\mathrm{A}} \cdot \tilde{p}=\lambda \cdot \tilde{p}
$$

d) As these fuzzy priorities must be ranked, they need to be translated into real numbers to make the ranking more obvious than fuzzy numbers. Several methods exist including the weighted average approach, the centre of area, the mean-max membership and the first (or last) of maxima. The most popular is the centre of area or centroid (Van Leekwijck \& Kerre, 1999).

Except for the fuzzy representation of the judgement scale, the steps of fuzzy AHP are the same as traditional AHP (Ishizaka \& Labib, 2011). Therefore, this paper will concentrate on the fuzzy membership function that represents the judgement scale. In the literature review, we found 27 different representations of fuzzy membership functions (Table 1), however none have been justified. Li and Kuo (2008) are the only one to ask the decision-maker to construct their own membership function but do not give any guidance on how to fulfil this task. This paper presents a new way to construct a personalised membership function. The methodology will be illustrated by a case study in banking.

\begin{tabular}{|c|c|c|c|c|}
\hline (A. Lee, Chen, & (Y.-L. Hsu, Lee, & (Alev Taskin, & (Büyüközkan \& & (H. Chen, Lee, \\
\& Chang, 2008; & \& Kreng, 2010; & 2009; M.-K. & Çifçi, 2012; Lo \& & \& Tong, 2007; \\
Paksoy, & Yuen \& Lau, & Chen \& Wang, & Wen, 2010) & Lu \& Wang, \\
Pehlivan, \& & $2011)$ & 2010; Chia- & & $2011)$ \\
Kahraman, & & Chi, 2010; S. & & \\
2012; Şen \& & & H. Hsu, Kao, \& & & \\
Çınar, 2010; & & Wu, 2009; Wu, & & \\
Zeydan, Çolpan, & & Lo, \& Hsu, & & \\
\& Çobanoğlu, & & $2008)$ & & \\
2011) & & & & $(1,1,3)$ \\
$(1,1,1)$ & $(1,1,1)$ & $(1,1,1)$ & $(1,1,2)$ & $(1,2,4)$ \\
$(1,2,3)$ & $(1,2,3)$ & $(1,2,3)$ & $(1,2,3)$ & $(1,3,5)$ \\
$(2,3,4)$ & $(2,3,4)$ & $(2,3,4)$ & $(2,3,4)$ & $(2,4,6)$ \\
$(3,4,5)$ & $(3,4,5)$ & $(3,4,5)$ & $(3,4,5)$ & $(4,5,7)$ \\
$(4,5,6)$ & $(4,5,6)$ & $(4,5,6)$ & $(4,5,6)$ & $(5,7,9)$ \\
$(5,6,7)$ & $(5,6,7)$ & $(5,6,7)$ & $(5,6,7)$ & $(6,8,9)$ \\
$(6,7,8)$ & $(6,7,8)$ & $(6,7,8)$ & $(6,7,8)$ & $(7,9,9)$ \\
$(7,8,9)$ & $(7,8,9)$ & $(7,8,9)$ & $(7,8,9)$ & \\
$(9,9,9)$ & $(8,9,9)$ & $(8,9,10)$ & $(8,9,10)$ & 3
\end{tabular}


[Preprint version, please cite as] Alessio Ishizaka, Nam Nguyen Hoang, Calibrated Fuzzy AHP for current bank account selection, Expert Systems with Applications, DOI 10.1016/j.eswa.2012.12.089, advance online publication

\begin{tabular}{|c|c|c|c|c|}
\hline $\begin{array}{l}\text { (Cho \& Lee, } \\
\text { 2011) }\end{array}$ & $\begin{array}{c}\text { (Javanbarg, } \\
\text { Scawthorn, } \\
\text { Kiyono, \& } \\
\text { Shahbodaghkhan, } \\
\text { 2012) }\end{array}$ & $\begin{array}{c}\text { (L.-C. Chen \& } \\
\text { Chu, 2012) }\end{array}$ & $\begin{array}{l}\text { (Özkır \& Demirel, } \\
\text { 2012) }\end{array}$ & $\begin{array}{c}\text { (Önüt, } \\
\text { Efendigil, \& } \\
\text { Soner Kara, } \\
\text { 2010) }\end{array}$ \\
\hline $\begin{array}{l}(0,1,2) \\
(1,2,3) \\
(2,3,4) \\
(3,4,5) \\
(4,5,6) \\
(5,6,7) \\
(6,7,8) \\
(7,8,9) \\
(8,9,9)\end{array}$ & $\begin{array}{c}(0.5,1,2) \\
(1,2,3) \\
(2,3,4) \\
(3,4,5) \\
(4,5,6) \\
(5,6,7) \\
(6,7,8) \\
(7,8,9) \\
(8,9,10)\end{array}$ & $\begin{array}{l}(1,1,2) \\
(1,2,3) \\
(2,3,4) \\
(3,4,5) \\
(4,5,6) \\
(5,6,7) \\
(6,7,8) \\
(7,8,9) \\
(8,9,9)\end{array}$ & $\begin{array}{c}(1,1,1) \text { or }(1,1,2) \\
(2,3,4) \\
(4,5,6) \\
(6,7,8) \\
(8,9,9) \\
\\
(1,1,1) \text { only if an } \\
\text { element is } \\
\text { compared with } \\
\text { itself, otherwise } \\
(1,1,2) \text { if the user } \\
\text { thinks they are } \\
\text { equal }\end{array}$ & $\begin{array}{c}(1,1,1) \text { or } \\
(1,1,3) \\
(1,3,5) \\
(3,5,7) \\
(5,7,9) \\
(7,9,9) \\
(1,1,1) \text { only if } \\
\text { an element is } \\
\text { compared with } \\
\text { itself, } \\
\text { otherwise } \\
(1,1,3) \text { if the } \\
\text { user thinks } \\
\text { they are equal }\end{array}$ \\
\hline $\begin{array}{c}\text { (Mentes \& } \\
\text { Helvacioglu, } \\
\text { 2012) }\end{array}$ & $\begin{array}{l}\text { (Bulut, Duru, } \\
\text { Keçeci, \& } \\
\text { Yoshida, 2012; } \\
\text { Cebeci, 2009; } \\
\text { Duru, Bulut, \& } \\
\text { Yoshida) }\end{array}$ & $\begin{array}{l}\text { (Haghighi, } \\
\text { Divandari, \& } \\
\text { Keimasi, 2010; } \\
\text { S.-H. Lee, } \\
\text { 2010) }\end{array}$ & $\begin{array}{c}\text { (Bozbura, } \\
\text { Beskese, \& } \\
\text { Kahraman, 2007; } \\
\text { Isaai, Kanani, } \\
\text { Tootoonchi, \& } \\
\text { Afzali, 2011; T.-C. } \\
\text { Wang \& Chen, } \\
\text { 2011) }\end{array}$ & $\begin{array}{l}\text { (Che, Wang, \& } \\
\text { Chuang, 2010) }\end{array}$ \\
\hline $\begin{array}{c}(1.00,1.00,1.25) \\
(1.25,1.50,1.75) \\
(1.75,2.00,2.25) \\
(2.25,2.50,2.75) \\
(2.75,3.00,3.00)\end{array}$ & $\begin{array}{l}(1,1,1) \\
(1,3,5) \\
(3,5,7) \\
(5,7,9) \\
(7,9,9)\end{array}$ & $\begin{array}{c}(1,1,1) \\
(1 / 2,1,3 / 2) \\
(1,3 / 2,2) \\
(3 / 2,2,5 / 2) \\
(2,5 / 2,3) \\
(5 / 2,3,7 / 2)\end{array}$ & $\begin{array}{l}(1.0,1.0,1.0) \\
(0.5,1.0,1.5) \\
(1.0,1.5,2.0) \\
(1.5,2.0,2.5) \\
(2.0,2.5,3.0) \\
(2.5,3.0,3.5)\end{array}$ & $\begin{array}{l}(1,1,1) \\
(1,2,3) \\
(2,3,4) \\
(3,4,5) \\
(4,5,6) \\
(5,6,7) \\
(6,7,8) \\
\end{array}$ \\
\hline $\begin{array}{c}\text { (Iç \& Yurdakul, } \\
\text { 2009) }\end{array}$ & (Hosang, 2011) & $\begin{array}{c}\text { (Seçme, } \\
\text { Bayrakdaroğlu, } \\
\text { \& Kahraman, } \\
\text { 2009) }\end{array}$ & $\begin{array}{l}\text { (Hadi-Vencheh \& } \\
\text { Mohamadghasemi, } \\
\text { 2011) }\end{array}$ & $\begin{array}{l}\text { (Nepal, Yadav, } \\
\text { \& Murat, } \\
\text { 2010) }\end{array}$ \\
\hline $\begin{array}{c}(1,1,1) \\
(2,3,4) \\
(4,5,6) \\
(6,7,8) \\
(8,9,10) \\
\end{array}$ & $\begin{array}{l}(1,1,2) \\
(1,3,5) \\
(3,5,7) \\
(5,7,9) \\
(8,9,9) \\
\end{array}$ & $\begin{array}{c}(1,1,1) \\
(2 / 3,1,3 / 2) \\
(1,3 / 2,2) \\
(3 / 2,2,5 / 2) \\
(5 / 2,3,7 / 2) \\
\end{array}$ & $\begin{array}{l}(1,1,2) \\
(1,2,3) \\
(2,3,4) \\
(3,4,5) \\
(4,5,5) \\
\end{array}$ & $\begin{array}{c}(1,1,3) \\
(1,3,5) \\
(3,5,7) \\
(5,7,9) \\
(7,9,11) \\
\end{array}$ \\
\hline $\begin{array}{l}\text { (Celik, Deha Er, } \\
\text { \& Ozok, 2009; } \\
\text { Kilincci \& Onal, }\end{array}$ & $\begin{array}{l}\text { (Büyüközkan, } \\
\text { Çifçi, \& } \\
\text { Güleryüz, 2011; }\end{array}$ & $\begin{array}{c}\text { (Cakir \& } \\
\text { Canbolat, } \\
\text { 2008; J. Wang, }\end{array}$ & $\begin{array}{c}\text { (Kaya \& } \\
\text { Kahraman, 2011a, } \\
\text { 2011c; Kutlu \& }\end{array}$ & $\begin{array}{c}\text { (Celik, } \\
\text { Kandakoglu, } \\
\text { \& Er, 2009; }\end{array}$ \\
\hline
\end{tabular}


[Preprint version, please cite as] Alessio Ishizaka, Nam Nguyen Hoang, Calibrated Fuzzy AHP for current bank account selection, Expert Systems with Applications, DOI 10.1016/j.eswa.2012.12.089, advance online publication

\begin{tabular}{|c|c|c|c|c|}
\hline $\begin{array}{c}2011 ; \text { Liu \& } \\
\text { Chen, 2009; } \\
\text { Rostamzadeh \& } \\
\text { Sofian, 2011) }\end{array}$ & $\begin{array}{c}\text { T.-S. Li \& } \\
\text { Huang, 2009) }\end{array}$ & $\begin{array}{c}\text { Fan, \& Wang, } \\
2010)\end{array}$ & $\begin{array}{c}\text { Ekmekçioğlu, } \\
2012)\end{array}$ & $\begin{array}{c}\text { Durán \& } \\
\text { Aguilo, 2008) }\end{array}$ \\
\hline$(1,1,1)$ & $(1,1,2)$ & $(1,1,2)$ & $(1,1,1)$ & $(1,1,3)$ \\
$(2 / 3,1,3 / 2)$ & $(2,3,4)$ & $(2,3,4)$ & $(1,1,1.5)$ & $(1,3,5)$ \\
$(3 / 2,2,5 / 2)$ & $(4,5,6)$ & $(4,5,6)$ & $(1,1.5,2)$ & $(3,5,7)$ \\
$(5 / 2,3,7 / 2)$ & $(6,7,8)$ & $(6,7,8)$ & $(1.5,2,2.5)$ & $(5,7,9)$ \\
$(7 / 2,4,9 / 2)$ & $(8,9,10)$ & $(8,9,9)$ & $(2,2.5,3)$ & $(7,9,9)$ \\
\hline$($ Kaya \& & $($ Iç \& Yurdakul, & $($ S. Li \& Kuo, & $($ Chiang \& Che, & \\
Kahraman, & $2009)$ & $2008)$ & $2010 ;$ Ho, 2012; & \\
$2011 b)$ & & & Ou, Fu, Hu, Chu, & \\
& & \& Chiou, 2011) & \\
$(1,1,1,1)$ & $(1,1,1,1)$ & Decision- & Not mentioned & \\
$(1,3 / 2,2,5 / 2)$ & $(2,3,4,5)$ & maker & & \\
$(3 / 2,2,5 / 2,3)$ & $(4,5,6,7)$ & constructs their & & \\
$(2,5 / 2,3,7 / 2)$ & $(6,7,8,9)$ & own & & \\
$(5 / 2,3,7 / 2,4)$ & $(8,9,10,10)$ & membership & & \\
& & function. & & \\
\hline
\end{tabular}

Table 1: Different definitions of membership function for the fuzzy scale

\section{Membership function calibration}

The calibration of the membership function is performed through a comparison of measurable alternatives. In our case, we used geometrical figures but it is possible for other items to be used (Figure 2). The participants were asked to compare their surface with the verbal scale given in Table 2. They were also informed that the figures were in an increasing order, so the questionnaire only had one scale direction (Table 3), e.g. A is necessarily smaller than B. Not all comparisons are required for the calibration; therefore only a subset was asked to avoid overwhelming the participants. The measured pairwise comparisons of the figures are given in Table 4. 
[Preprint version, please cite as] Alessio Ishizaka, Nam Nguyen Hoang, Calibrated Fuzzy AHP for current bank account selection, Expert Systems with Applications, DOI 10.1016/j.eswa.2012.12.089, advance online publication

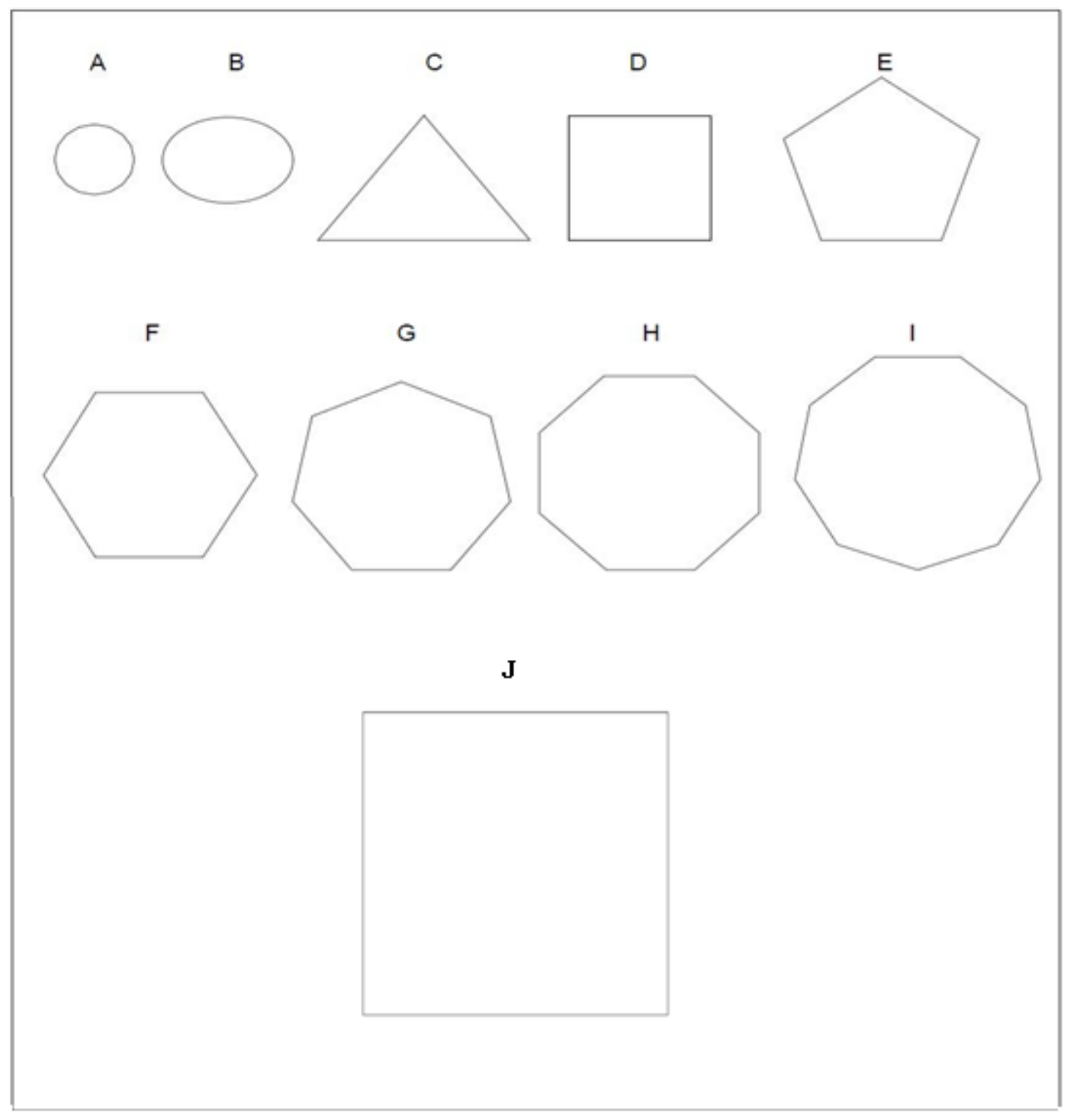

Figure 2: Geometrical figures

\begin{tabular}{|c|c|}
\hline Abbreviation & Definition \\
\hline Equ & Equal size \\
\hline Eq/mod & Equal to Moderately different \\
\hline Moderate & Moderately different \\
\hline Mod/Str & Moderately to strongly different \\
\hline Strong & Strongly different \\
\hline Str/verStr & Strongly to very strongly different \\
\hline Ver Str & Very strongly different \\
\hline verStr/Extr & Very strongly to extremely different \\
\hline Ext & Extremely different \\
\hline
\end{tabular}

Table 2: Evaluation scale 
[Preprint version, please cite as] Alessio Ishizaka, Nam Nguyen Hoang, Calibrated Fuzzy AHP for current bank account selection, Expert Systems with Applications, DOI 10.1016/j.eswa.2012.12.089, advance online publication

\begin{tabular}{|c|c|c|c|c|c|c|c|c|c|c|}
\hline Figure I & \multicolumn{9}{|c|}{ How much Figure I is bigger than Figure II? } & Figure II \\
\hline $\mathrm{B}$ & Equal & Eq/mode & moderate & $\mathrm{mod} / \mathrm{str}$ & strong & str/verstr & verstr & verstr/ext & Extreme & A \\
\hline C & Equal & Eq/mode & moderate & $\mathrm{mod} / \mathrm{str}$ & strong & str/verstr & verstr & verstr/ext & Extreme & A \\
\hline $\mathrm{D}$ & Equal & Eq/mode & moderate & $\mathrm{mod} / \mathrm{str}$ & strong & str/verstr & verstr & verstr/ext & Extreme & A \\
\hline $\mathrm{E}$ & Equal & Eq/mode & moderate & $\mathrm{mod} / \mathrm{str}$ & strong & str/verstr & verstr & verstr/ext & Extreme & A \\
\hline $\mathrm{F}$ & Equal & $\mathrm{Eq} /$ mode & moderate & $\mathrm{mod} / \mathrm{str}$ & strong & str/verstr & verstr & verstr/ext & Extreme & $A$ \\
\hline $\mathrm{G}$ & Equal & $\mathrm{Eq} /$ mode & moderate & $\mathrm{mod} / \mathrm{str}$ & strong & str/verstr & verstr & verstr/ext & Extreme & $A$ \\
\hline $\mathrm{H}$ & Equal & Eq/mode & moderate & $\mathrm{mod} / \mathrm{str}$ & strong & str/verstr & verstr & verstr/ext & Extreme & A \\
\hline 1 & Equal & $\mathrm{Eq} / \mathrm{mode}$ & moderate & $\mathrm{mod} / \mathrm{str}$ & strong & str/verstr & verstr & verstr/ext & Extreme & $A$ \\
\hline
\end{tabular}

Table 3: Extract of the questionnaire

\begin{tabular}{|c|c|c|c|c|c|c|c|c|c|c|}
\hline & $\mathrm{A}$ & $\mathrm{B}$ & $\mathrm{C}$ & $\mathrm{D}$ & $\mathrm{E}$ & $\mathrm{F}$ & $\mathrm{G}$ & $\mathrm{H}$ & $\mathrm{I}$ & $\mathrm{J}$ \\
\hline $\mathrm{A}$ & & & & & & & & & & \\
\hline $\mathrm{B}$ & 2 & & & & & & & & & \\
\hline $\mathrm{C}$ & 3 & $3 / 2$ & & & & & & & & \\
\hline $\mathrm{D}$ & 4 & $4 / 2$ & $4 / 3$ & & & & & & & \\
\hline $\mathrm{E}$ & 5 & $5 / 2$ & $5 / 3$ & $5 / 4$ & & & & & & \\
\hline $\mathrm{F}$ & 6 & $6 / 2$ & $6 / 3$ & $6 / 4$ & $6 / 5$ & & & & & \\
\hline $\mathrm{G}$ & 7 & $7 / 2$ & $7 / 3$ & $7 / 4$ & $7 / 5$ & $7 / 6$ & & & & \\
\hline $\mathrm{H}$ & 8 & $8 / 2$ & $8 / 3$ & $8 / 4$ & $8 / 5$ & $8 / 6$ & $8 / 7$ & & & \\
\hline $\mathrm{I}$ & 9 & $9 / 2$ & $9 / 3$ & $9 / 4$ & $9 / 5$ & $9 / 6$ & $9 / 7$ & $9 / 8$ & & \\
\hline $\mathrm{J}$ & & $10 / 2$ & $10 / 3$ & $10 / 4$ & $10 / 5$ & & & & & \\
\hline
\end{tabular}

Table 4: Real measured pairwise comparisons; comparison is not done for empty squares

The verbal judgements (Table 3 ) given by the decision-maker are matched with the real values (Table 4). For example, suppose that the decision-maker evaluates a "very strong" difference between figures $\mathrm{G}$ and $\mathrm{A}, \mathrm{D}$ and $\mathrm{A}$ and also between figure I and $\mathrm{B}$. The real values of these three evaluations (i.e. 7, 4, 4.5) are entered into the matching table (Table 5). Therefore, it can be deduced that the decision maker values outcomes of between 4.5 and 7 as "very strong".

All the judgements matched with the real measures are entered into a table (Table 5). For each verbal judgement, the minimal mean and maximal values are calculated. They correspond to the angle points of the customised membership function. Figure 3 represents the customised membership functions of all verbal judgements. Notice that these membership functions are not similar (e.g. the wideness of the membership function "very strong" is much larger than "moderate") because they depend on the person's interpretation of verbal judgements. 
[Preprint version, please cite as] Alessio Ishizaka, Nam Nguyen Hoang, Calibrated Fuzzy AHP for current bank account selection, Expert Systems with Applications, DOI 10.1016/j.eswa.2012.12.089, advance online publication

\begin{tabular}{|c|c|c|c|c|c|c|c|c|c|}
\hline Scale & Equal & $\begin{array}{c}\text { Eq/ } \\
\text { Mod }\end{array}$ & Moderate & $\begin{array}{l}\text { Mod/ } \\
\text { Str }\end{array}$ & Strong & $\begin{array}{c}\text { Str/ } \\
\text { very Str }\end{array}$ & $\begin{array}{c}\text { Very } \\
\text { Strong }\end{array}$ & $\begin{array}{r}\text { Ver str/ } \\
\text { extreme }\end{array}$ & Extreme \\
\hline \multirow{9}{*}{ 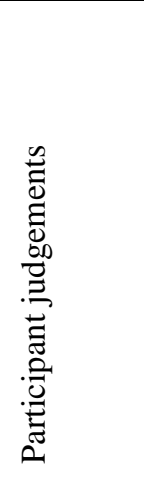 } & 1.20 & 2.00 & 3.00 & 4.00 & 5.00 & 6.00 & 7.00 & 8.00 & 9.00 \\
\hline & 1.00 & 1.33 & 1.50 & 2.33 & 2.50 & 3.00 & 4.00 & 4.50 & \\
\hline & & 1.40 & 2.00 & 1.50 & 2.67 & 3.50 & 4.50 & 9.00 & \\
\hline & & 1.60 & 1.67 & 1.75 & 2.67 & 3.60 & & 6.00 & \\
\hline & & 1.17 & 2.00 & 2.00 & 3.00 & & & & \\
\hline & & 1.33 & 1.25 & 1.50 & 2.25 & & & & \\
\hline & & 1.14 & 1.80 & & & & & & \\
\hline & & 1.29 & & & & & & & \\
\hline & & 1.13 & & & & & & & \\
\hline$p(\min )$ & 1.00 & 1.13 & 1.25 & 1.50 & 2.25 & 3.00 & 4.00 & 4.50 & 9.00 \\
\hline p(mean) & 1.10 & 1.38 & 1.89 & 2.18 & 3.01 & 4.03 & 5.17 & 6.88 & 9.00 \\
\hline $\mathbf{p}(\max )$ & 1.20 & 2.00 & 3.00 & 4.00 & 5.00 & 6.00 & 7.00 & 9.00 & 9.00 \\
\hline
\end{tabular}

Table 5: Matching table

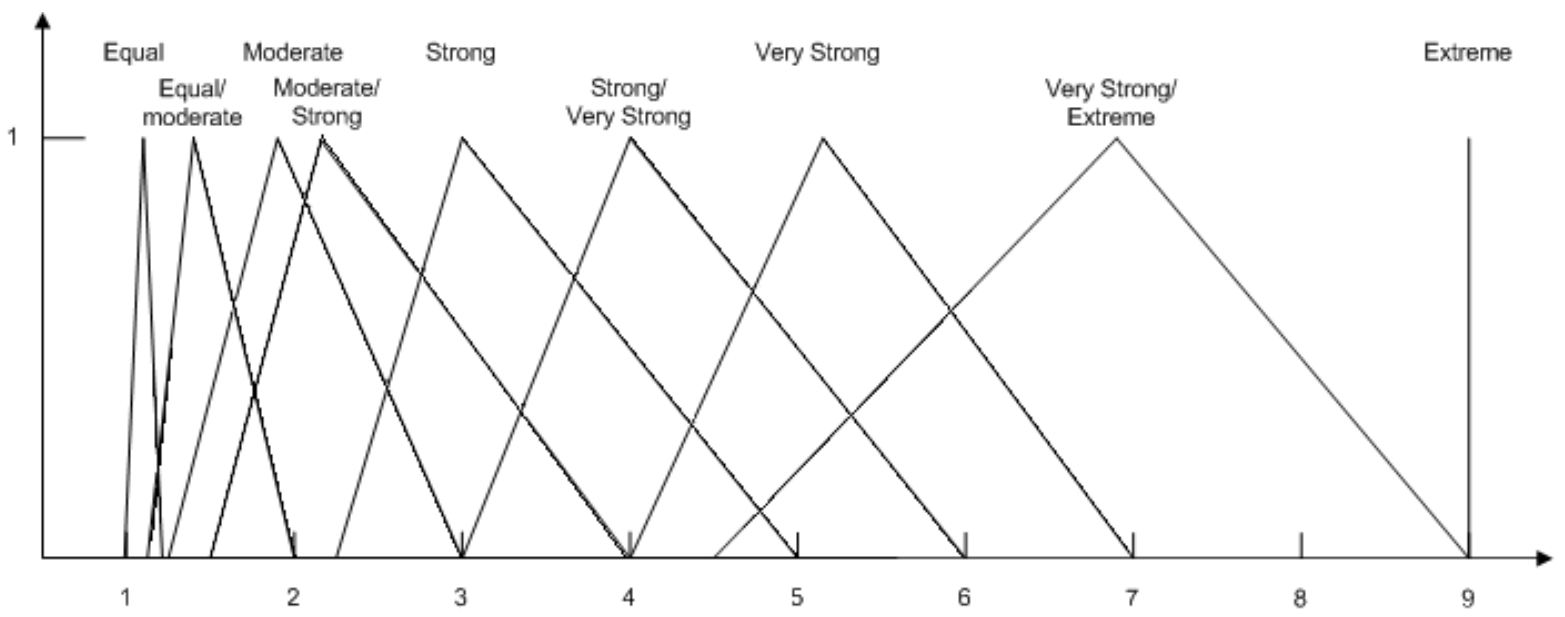

Figure 3: Customised membership functions

\section{Case study}

\subsection{Introduction}

The development of an appealing product may have a long-term impact on the profitability of companies. This is especially true in the banking sector, where students often remain with the same bank when they leave education. Students are not a profitable segment of the market because their income is low, however they are the potentially high earner in the future. As a result, it is in the best interests of the bank to attract and retain these customers early.

This explorative study will give an insight into the most important criteria in selecting a student bank account using calibrated fuzzy AHP, described in section 3. 
[Preprint version, please cite as] Alessio Ishizaka, Nam Nguyen Hoang, Calibrated Fuzzy AHP for current bank account selection, Expert Systems with Applications, DOI 10.1016/j.eswa.2012.12.089, advance online publication

\subsection{Criteria description}

In the literature there are several studies for bank selection in different countries: Romania (Katircioglu, Tumer, \& Kılınç, 2011); Ghana (Hinson, Owusu-Frimpong, \& Dasah, 2011; Mahmoud, Tweneboah-Koduah, \& Danku, 2011); USA (J. Lee \& Marlowe, 2003), Northern Cyprus (Katircioglu, Unlucan, \& Dalci, 2011; Safakli, 2007); Malaysia (Ahmad, Rustam, \& Dent, 2011; Amin, 2008; Mokhlis, Salleh, \& Mat, 2011); Greece (Lymperopoulos, Chaniotakis, \& Soureli, 2006); Bahrain (Al-Ajmi, Abo Hussain, \& Al-Saleh, 2009; Almossawi, 2001); United Kingdom (Devlin \& Gerrard, 2005; Farquhar \& Panther, 2008; Thwaitesa \& Verea, 1995); Singapore (Ta \& Har, 2000), Poland (Kennington, Hill, \& Rakowska, 1996); Hong Kong (Denton \& Chan, 1991); India (Gupta \& Dev, 2012). Each study has its own list of criteria. As the utilisation of AHP becomes difficult with a large number of criteria, similar factors were grouped together (Table 6) and structured into a hierarchy (Figure 4). This also avoids the problem of overweighting dependent criteria (e.g. internal and external bank appearance).

Some criteria have not been considered because:

- They are out-dated, for example, ATM service. Banks have a consensus scheme to share ATM information systems, therefore; a person can withdraw cash either free of charge or for a small fee from any ATM belonging to another bank.

- They are outside the control of the banks, such as recommendations from friends and relatives. Some studies also suggest that these criteria are negligible in bank account selection (Almossawi, 2001; Ta \& Har, 2000).

\begin{tabular}{|c|c|}
\hline $\begin{array}{l}\text { Services } \\
\text { Intangible provisions } \\
\text { to the customer define } \\
\text { the bank services. }\end{array}$ & $\begin{array}{l}\text { Personnel service quality: efficiency and competence (Al-Ajmi, } \\
\text { et al., 2009; Blankson, et al., 2009; Gupta \& Dev, 2012; Hinson, } \\
\text { et al., 2011; Katircioglu, Tumer, et al., 2011; Laroche, et al., } \\
\text { 1986; Lymperopoulos, et al., 2006; Manrai \& Manrai, 2007; } \\
\text { Mokhlis, et al., 2011; Safakli, 2007; Thwaitesa \& Verea, 1995) } \\
\text { and consistency (Lymperopoulos, et al., 2006) of the personnel, } \\
\text { staff friendliness (Al-Ajmi, et al., 2009; Almossawi, 2001; } \\
\text { Hinson, et al., 2011; Laroche, et al., 1986; Mahmoud, et al., } \\
\text { 2011; Safakli, 2007), speed of the service (Blankson, et al., 2009; } \\
\text { Gupta \& Dev, 2012; Katircioglu, Tumer, et al., 2011; } \\
\text { Lymperopoulos, et al., 2006; Thwaitesa \& Verea, 1995) }\end{array}$ \\
\hline & $\begin{array}{l}\text { - Banking service features: Card type (cash, debit or credit card) } \\
\text { with favourable conditions (Katircioglu, Tumer, et al., 2011), } \\
\text { accessibility to the account (internet banking, phone banking) }\end{array}$ \\
\hline
\end{tabular}


[Preprint version, please cite as] Alessio Ishizaka, Nam Nguyen Hoang, Calibrated Fuzzy AHP for current bank account selection, Expert Systems with Applications, DOI 10.1016/j.eswa.2012.12.089, advance online publication

\begin{tabular}{|c|c|c|}
\hline & & $\begin{array}{l}\text { (Al-Ajmi, et al., 2009; Almossawi, 2001; Blankson, et al., 2009; } \\
\text { Devlin \& Gerrard, 2005; Katircioglu, Tumer, et al., 2011; } \\
\text { Mahmoud, et al., 2011; Ta \& Har, 2000), ease of opening a } \\
\text { current account (Almossawi, 2001), hours of operations (Al- } \\
\text { Ajmi, et al., 2009; Almossawi, 2001; Devlin \& Gerrard, 2005; } \\
\text { Gupta \& Dev, 2012; Kamvysi, et al., 2010; Katircioglu, Tumer, } \\
\text { et al., 2011; Kennington, et al., 1996; Laroche, et al., 1986; } \\
\text { Manrai \& Manrai, 2007; Ta \& Har, 2000), international funds } \\
\text { transfer (Mahmoud, et al., 2011) }\end{array}$ \\
\hline & & $\begin{array}{l}\text { Building quality: Branch location (Al-Ajmi, et al., 2009; } \\
\text { Almossawi, 2001; Blankson, Omar, \& Cheng, 2009; Devlin \& } \\
\text { Gerrard, 2005; Gupta \& Dev, 2012; Kamvysi, Gotzamani, } \\
\text { Georgiou, \& Andronikidis, 2010; Katircioglu, Tumer, et al., } \\
\text { 2011; J. Lee \& Marlowe, 2003; Lewis, 1981; Mahmoud, et al., } \\
\text { 2011; Mokhlis, et al., 2011; Safakli, 2007; Thwaitesa \& Verea, } \\
\text { 1995), parking facilities and accessibility (Al-Ajmi, et al., 2009; } \\
\text { Almossawi, 2001; Gupta \& Dev, 2012; Kamvysi, et al., 2010; } \\
\text { Laroche, Rosenblatt, \& Manning, 1986; Mahmoud, et al., 2011; } \\
\text { Safakli, 2007), external bank appearance (Hinson, et al., 2011; } \\
\text { Katircioglu, Tumer, et al., 2011; Manrai \& Manrai, 2007; Safakli, } \\
\text { 2007), bank decor and atmosphere (e.g. waiting lounge, drinking } \\
\text { water) (Al-Ajmi, et al., 2009; Gupta \& Dev, 2012; Katircioglu, } \\
\text { Tumer, et al., 2011; Manrai \& Manrai, 2007; Mokhlis, et al., } \\
\text { 2011) }\end{array}$ \\
\hline \multirow[t]{2}{*}{$\begin{array}{l}\text { Financial Factors } \\
\text { The financial factors } \\
\text { are defined by a direct } \\
\text { monetary benefit. }\end{array}$} & - & $\begin{array}{l}\text { Charges: Low fees or charges (Almossawi, 2001; Blankson, et } \\
\text { al., 2009; Devlin \& Gerrard, 2005; Gupta \& Dev, 2012; } \\
\text { Kamvysi, et al., 2010; Katircioglu, Tumer, et al., 2011; J. Lee \& } \\
\text { Marlowe, 2003; Lymperopoulos, et al., 2006; Mokhlis, et al., } \\
\text { 2011; Thwaitesa \& Verea, 1995) }\end{array}$ \\
\hline & & $\begin{array}{l}\text { Interest rates: High interest rates (Al-Ajmi, et al., 2009; } \\
\text { Almossawi, 2001; Devlin \& Gerrard, 2005; Gupta \& Dev, 2012; } \\
\text { Kamvysi, et al., 2010; Katircioglu, Tumer, et al., 2011; }\end{array}$ \\
\hline
\end{tabular}


[Preprint version, please cite as] Alessio Ishizaka, Nam Nguyen Hoang, Calibrated Fuzzy AHP for current bank account selection, Expert Systems with Applications, DOI 10.1016/j.eswa.2012.12.089, advance online publication

\begin{tabular}{|c|c|}
\hline & $\begin{array}{l}\text { Kennington, et al., 1996; J. Lee \& Marlowe, 2003; } \\
\text { Lymperopoulos, et al., 2006; Manrai \& Manrai, 2007; Ta \& Har, } \\
\text { 2000). }\end{array}$ \\
\hline & $\begin{array}{l}\text { - Overdraft Facilities: Overdraft availability and size (Kamvysi, } \\
\text { et al., 2010; Lewis, 1981; Thwaitesa \& Verea, 1995) }\end{array}$ \\
\hline $\begin{array}{l}\text { Extra benefits } \\
\text { Extra benefits given } \\
\text { by the bank when } \\
\text { opening an account. }\end{array}$ & $\begin{array}{l}\text { - Bonuses: A one-off gift given when opening or switching } \\
\text { accounts (Katircioglu, Tumer, et al., 2011; Lewis, 1981; Mokhlis, } \\
\text { et al., 2011). It could be a cash-back offer (Blankson, et al., } \\
\text { 2009), or music to download, a SIM card, an iPod, etc. }\end{array}$ \\
\hline $\begin{array}{l}\text { to the students' life } \\
\text { offering things such } \\
\text { as travel discounts } \\
\text { and laptop vouchers. }\end{array}$ & $\begin{array}{l}\text { - Incentives: Incentives are available as long as the account is } \\
\text { open (Devlin \& Gerrard, 2005). This includes mobile or car } \\
\text { insurance, extra top-ups on mobile phones, discounts on travel or } \\
\text { shopping, discounted international payments, etc. }\end{array}$ \\
\hline
\end{tabular}

Table 6: Bank selection criteria

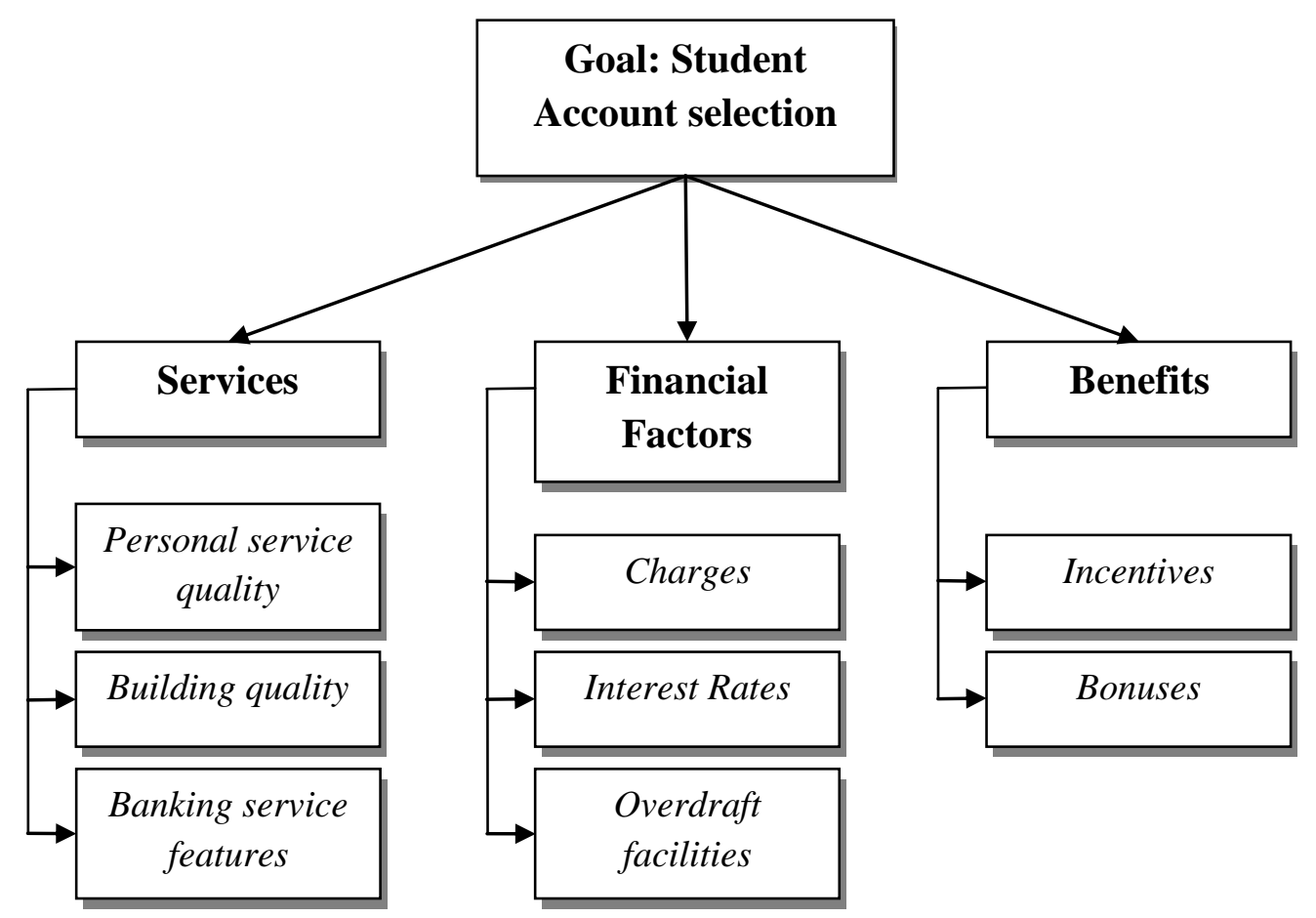

Figure 4: Hierarchy of the criteria 
[Preprint version, please cite as] Alessio Ishizaka, Nam Nguyen Hoang, Calibrated Fuzzy AHP for current bank account selection, Expert Systems with Applications, DOI 10.1016/j.eswa.2012.12.089, advance online publication

\subsection{Demography of the participants}

Forty participants of the University of Portsmouth were recruited in a sample of equal gender and nationality proportions (Table 7).

Table 7: Sample selection of the study

\begin{tabular}{|l|c|c|}
\hline & Male & Female \\
\hline British student & 10 & 10 \\
\hline International student & 10 & 10 \\
\hline
\end{tabular}

Participants are aged between 19 and 30 (Table 8). Twenty-three students are on a bachelor course and seventeen on a masters level course. Only participant P36 had full-time work experience of more than six months.

Table 8: Demography of the participants

\begin{tabular}{|c|l|c|c|c||}
\hline$\#$ & Course & Age & Gender & Nationality \\
\hline \hline P1 & MsC Finance & 27 & M & British \\
\hline P2 & BA Accounting and Finance & 19 & F & Vietnamese \\
\hline P3 & MsC Business and Management & 24 & F & Indian \\
\hline P4 & MA Marketing & 24 & F & Thailand \\
\hline P5 & MsC Business and Management & 23 & F & Vietnamese \\
\hline P6 & BsC Business and Economic & 20 & F & British \\
\hline P7 & MsC Business and Management & 25 & M & Vietnamese \\
\hline P8 & BsC Biology & 21 & M & British \\
\hline P9 & BA Accountancy and Financial Management & 20 & F & British \\
\hline P10 & BA Computing & 21 & F & British \\
\hline P11 & BsC Crime and Criminology & 24 & M & British \\
\hline P12 & MsC Financial Decision Analysis & 23 & M & Vietnamese \\
\hline P13 & BA Digital Marketing & 22 & F & British \\
\hline P14 & MsC Financial Decision Analysis & 25 & M & Malaysian \\
\hline P15 & BsC Digital Forensics & 22 & M & British \\
\hline P16 & MsC Finance & 26 & M & Chinese \\
\hline P17 & MsC Construction Project Management & 27 & M & British \\
\hline P18 & BA Education and Training studies & 22 & F & British \\
\hline P19 & BA English Literature & 20 & F & Chinese \\
\hline P20 & BA Business Administration & 19 & M & Malaysian \\
\hline P21 & MsC Forensic Accounting & 25 & M & Chinese \\
\hline P22 & BA Business Enterprise & 20 & M & Indian \\
\hline P23 & BA Accounting and Business & 21 & M & Vietnamese \\
\hline P24 & MsC Finance & Chinese \\
\hline
\end{tabular}


[Preprint version, please cite as] Alessio Ishizaka, Nam Nguyen Hoang, Calibrated Fuzzy AHP for current bank account selection, Expert Systems with Applications, DOI 10.1016/j.eswa.2012.12.089, advance online publication

\begin{tabular}{|c|l|c|c|c||}
\hline P25 & MsC Business and Management & 25 & F & British \\
\hline P26 & BA Business with Business Communication & 20 & M & Vietnamese \\
\hline P27 & MsC Finance & 23 & M & British \\
\hline P28 & FdA Policy Studies & 26 & M & British \\
\hline P29 & BsC Web and Game Technology & 20 & M & British \\
\hline P30 & MsC Finance & 27 & M & British \\
\hline P31 & BsC Accounting and Finance & 20 & M & British \\
\hline P32 & MsC Finance & 23 & F & Chinese \\
\hline P33 & MsC Business and Economic & 24 & F & British \\
\hline P34 & MsC Business and Management & 23 & F & Vietnamese \\
\hline P35 & MsC Finance & 25 & F & Vietnamese \\
\hline P36 & MsC Human Resource Management & 30 & F & British \\
\hline P37 & MA Marketing & 25 & F & Vietnamese \\
\hline P38 & MsC Finance & 25 & F & British \\
\hline P39 & MA Sale Management & 24 & F & British \\
\hline P40 & MsC Coach and Development & 26 & F & Chinese \\
\hline
\end{tabular}

\subsection{Questionnaire collection mode}

To increase the response rate, different collection channels were used:

- E-mail: This collection mode has a low associated cost (no printing and postage) and is timesaving as a large population can be targeted at once. The questionnaire was sent to seventy-five students. Twenty-five questionnaires were returned but only fourteen were correctly completed. The perceived disadvantage of this collection mode is the relatively low response rate (33\%) and the delays between the dates the email was sent and getting a reply.

- Face-to-face communication: This channel allows interaction with the participants, in particular, the relevance of the study can be emphasised and instructions can be repeated if necessary. Forty people were approached, thirty agreed to complete the questionnaire (65\% response rate) but only nineteen were fit for use. This collection mode is more time consuming than the other methods.

- Social network: This channel has a large outreach, low cost and is timesaving. The questionnaire was posted onto the university Facebook page, however very few responses were received (twenty) and only seven could be used. This was the lowest usable rate of the three channels (Table 9). 
[Preprint version, please cite as] Alessio Ishizaka, Nam Nguyen Hoang, Calibrated Fuzzy AHP for current bank account selection, Expert Systems with Applications, DOI 10.1016/j.eswa.2012.12.089, advance online publication

Table 9: Modes of data collection

\begin{tabular}{l|l|l|l|l|l}
\hline \hline $\begin{array}{c}\text { Mode of } \\
\text { communication }\end{array}$ & $\begin{array}{l}\text { Questionnaire } \\
\text { distributed }\end{array}$ & $\begin{array}{c}\mathbf{N}^{\mathbf{0}} \text { of } \\
\text { responses }\end{array}$ & $\begin{array}{c}\mathbf{N}^{\mathbf{0}} \text { of usable } \\
\text { responses }\end{array}$ & $\begin{array}{c}\text { Responses } \\
\text { rate }\end{array}$ & $\begin{array}{c}\text { Usable } \\
\text { response rate }\end{array}$ \\
\hline \hline Email & 75 & 25 & 14 & $33 \%$ & $56 \%$ \\
Face to face & 40 & 30 & 19 & $75 \%$ & $63.33 \%$ \\
Social network & High & 20 & 7 & (Very low) & $35 \%$ \\
\hline Total & $\mathbf{1 1 5}$ to high & $\mathbf{7 5}$ & $\mathbf{4 0}$ & $\mathbf{n} / \mathbf{a}$ & $\mathbf{n} / \mathbf{a}$ \\
\hline \hline
\end{tabular}

A questionnaire is considered valid if fully completed and has consistent pairwise comparisons. In this study, twenty questionnaires were incomplete and fifteen were inconsistent.

For each participant, a matching table (e.g. Table 5) has been constructed and a customised scale has been calculated. The results were then imported into Expert Choice, where priorities were obtained with Fuzzy AHP and aggregated in a group decision using the geometric mean.

\section{5. $\quad$ Results}

Table 10 summaries the importance of the criteria for selecting a current account. The average consistency is very high, which indicates that the participants have a clear view of their priorities.

These results support previous studies highlighting the importance of services in bank account selection, in particular the personal service quality. The second most important criterion was bonuses, which proved to be more attractive than long-term incentives. Financial factors have the lowest score. This may be explained by the low income of students and their inability to invest.

The British and international students (Comparison I, Table 10) had very similar scores. International students scored slightly higher for the criterion services and slightly lower for financial factors. Being unfamiliar with British banking systems, it is understandable that they would prefer a good personal service with tailored explanations. The employees should also understand their particular needs. Financial factors were not so important as they tend to leave the country after graduation. Their bank accounts are used as a deposit base rather than an investment instrument. It is also not surprising that they prefer immediate bonus rather than incentives that stop with the closure of the account.

The female and male expectations are slightly different (Comparison II, Table 10). Females have a slight preference for better services and financial factors, whilst males prefer the benefits criterion. 
[Preprint version, please cite as] Alessio Ishizaka, Nam Nguyen Hoang, Calibrated Fuzzy AHP for current bank account selection, Expert Systems with Applications, DOI 10.1016/j.eswa.2012.12.089, advance online publication

Table 10: Importance of criteria for current account selection

\begin{tabular}{|c|c|c|c|c|c|c|c|}
\hline Comparisons & Overall & & rison I & Com & rison II & Comp & ison III \\
\hline Criteria & & Home & $\begin{array}{l}\text { Inte- } \\
\text { rnational }\end{array}$ & Females & Males & $\begin{array}{l}\text { Under- } \\
\text { graduate }\end{array}$ & $\begin{array}{l}\text { Post- } \\
\text { graduate }\end{array}$ \\
\hline Number of participants & 40 & 20 & 20 & 20 & 20 & 17 & 23 \\
\hline Services & $44.5 \%$ & $42.5 \%$ & $46.3 \%$ & $46.5 \%$ & $42.2 \%$ & $40.6 \%$ & $47.1 \%$ \\
\hline Personal service quality & $19.9 \%$ & $19.3 \%$ & $20.4 \%$ & $20.9 \%$ & $18.7 \%$ & $17.9 \%$ & $21.3 \%$ \\
\hline Building quality & $11.1 \%$ & $9.5 \%$ & $12.8 \%$ & $10.9 \%$ & $11.2 \%$ & $10.8 \%$ & $11.1 \%$ \\
\hline Banking service features & $13.5 \%$ & $13.8 \%$ & $13.2 \%$ & $14.8 \%$ & $12.2 \%$ & $11.9 \%$ & $14.7 \%$ \\
\hline Financial Factors & $22.1 \%$ & $24.0 \%$ & $20.3 \%$ & $24.3 \%$ & $20.0 \%$ & $20.4 \%$ & $23.3 \%$ \\
\hline Charges & $8.7 \%$ & $9.2 \%$ & $8.3 \%$ & $9.7 \%$ & $7.8 \%$ & $8.8 \%$ & $8.6 \%$ \\
\hline Interest rates & $6.5 \%$ & $7.6 \%$ & $5.5 \%$ & $7.5 \%$ & $5.6 \%$ & $5.8 \%$ & $6.9 \%$ \\
\hline Overdraft facilities & $6.9 \%$ & $7.3 \%$ & $6.5 \%$ & $7.2 \%$ & $6.6 \%$ & $5.8 \%$ & $7.7 \%$ \\
\hline Benefits & $33.4 \%$ & $33.4 \%$ & $33.4 \%$ & $29.3 \%$ & $37.9 \%$ & $39.0 \%$ & $29.6 \%$ \\
\hline Bonuses & $18.6 \%$ & $17.5 \%$ & $19.7 \%$ & $16.5 \%$ & $20.8 \%$ & $20.9 \%$ & $\% 16.9$ \\
\hline Incentives & $14.8 \%$ & $16.0 \%$ & $13.6 \%$ & $12.7 \%$ & $17.1 \%$ & $18.0 \%$ & $\% 12.7$ \\
\hline Inconsistency rate & $0 \%$ & $0 \%$ & $0 \%$ & $0 \%$ & $0 \%$ & $0 \%$ & $0 \%$ \\
\hline
\end{tabular}

Undergraduate students rate the Benefits criterion very highly (39\%), almost as high as the services (40.6\%) (Comparison III, Table 10). Postgraduates prefer the services by almost half of the weight $(47.1 \%)$.

\section{Conclusion}

The contribution of this study can be viewed from both a theoretical and practical dimension. Theoretically, this paper presents a new customised fuzzy-AHP method. Practically, it provides insight into criteria selection for bank accounts among students.

When data are precisely known, they can be represented with crisps numbers. However, in most real-world situations, data cannot be assessed precisely because it is unquantifiable, incomplete, undisclosed or vague. Decision-makers are often uncertain in assigning the evaluation with conventional AHP, therefore a hybrid multi-criteria technique is used. Linguistic terms have been introduced to better mirror the vagueness and subjectivity of the evaluation. These are then translated into numerical values with fuzzy formats described by membership functions. In previous work, the construction of membership functions was never discussed and was the same for every decision-maker. In this paper, a new process to calibrate these functions according to the decision-maker's subjectivity is proposed. This 
[Preprint version, please cite as] Alessio Ishizaka, Nam Nguyen Hoang, Calibrated Fuzzy AHP for current bank account selection, Expert Systems with Applications, DOI 10.1016/j.eswa.2012.12.089, advance online publication

significantly contributes to more precise and customised results. The methodology provides an effective tool for evaluating fuzzy representations and for modelling subjective and ambiguous situations. Due to the customisation of each personal verbal evaluation, the tool's adaptability is enhanced for multiple criteria group decision-making problems in a 'fuzzy' situation.

To illustrate this new customised fuzzy-AHP method, a study on the importance of the criteria for selecting a bank account for students was conducted. As the research was for illustrative purposes, it should be considered explorative. The sample was small but some conclusions were drawn nevertheless. The face-to-face collection of data was the most effective collection method, with a much higher response rate and consistency index. The study highlighted once more in the academic literature the importance of service in the banking sector and found that financial factors were less important. Bonuses were more attractive to international students and therefore; it is not surprising that several UK banks prefer to offer bonuses than attractive financial conditions. It is essential to understand the preference of the consumer and to have a strategic design in place to meet their expectations. The findings of the study serve as a starting point for bank managers to understand the importance of the selection criteria, however, further studies on a larger scale are needed to confirm these observations.

On the methodological part, the fuzzy calibration methodology is very versatile and flexible in its areas of applications and integration with other methods. Future studies could combine the fuzzy calibration methodology with other MCDA techniques, such as TOPSIS, PROEMTHEE and ELECTRE.

\section{REFERENCES}

Ahmad, K., Rustam, G., \& Dent, M. (2011). Brand preference in Islamic banking. Journal of Islamic Marketing, 2, 74 - 82.

Al-Ajmi, J., Abo Hussain, H., \& Al-Saleh, N. (2009). Clients of conventional and Islamic banks in Bahrain: How they choose which bank to patronize. International Journal of Social Economics, 36, 1086 - 1112.

Alev Taskin, G. (2009). Evaluation of hazardous waste transportation firms by using a two step fuzzy-AHP and TOPSIS methodology. Expert Systems with Applications, 36, 4067-4074.

Almossawi, M. (2001). Bank selection criteria employed by college students in Bahrain: an empirical analysis. International Journal of Bank Marketing, 19, 115 - 125.

Amin, H. (2008). Choice criteria for Islamic home financing: Empirical investigation among Malaysian bank customers. International Journal of Housing Markets and Analysis, 1, $256-274$.

Blankson, C., Omar, O., \& Cheng, J. M.-S. (2009). Retail bank selection in developed and developing countries: A cross-national study of students' bank-selection criteria. Thunderbird International Business Review, 51, 183-198. 
[Preprint version, please cite as] Alessio Ishizaka, Nam Nguyen Hoang, Calibrated Fuzzy AHP for current bank account selection, Expert Systems with Applications, DOI 10.1016/j.eswa.2012.12.089, advance online publication

Bozbura, F., Beskese, A., \& Kahraman, C. (2007). Prioritization of human capital measurement indicators using fuzzy AHP. Expert Systems with Applications, 32, $1100-1112$.

Bulut, E., Duru, O., Keçeci, T., \& Yoshida, S. (2012). Use of consistency index, expert prioritization and direct numerical inputs for generic fuzzy-AHP modeling: A process model for shipping asset management. Expert Systems with Applications, 39, 19111923.

Büyüközkan, G., \& Çifçi, G. (2012). A combined fuzzy AHP and fuzzy TOPSIS based strategic analysis of electronic service quality in healthcare industry. Expert Systems with Applications, 39, 2341-2354.

Büyüközkan, G., Çifçi, G., \& Güleryüz, S. (2011). Strategic analysis of healthcare service quality using fuzzy AHP methodology. Expert Systems with Applications, 38, 94079424.

Cakir, O., \& Canbolat, M. (2008). A web-based decision support system for multi-criteria inventory classification using fuzzy AHP methodology. Expert Systems with Applications, 35, 1367-1378.

Cebeci, U. (2009). Fuzzy AHP-based decision support system for selecting ERP systems in textile industry by using balanced scorecard. Expert Systems with Applications, 36, 8900-8909.

Celik, M., Deha Er, I., \& Ozok, A. (2009). Application of fuzzy extended AHP methodology on shipping registry selection: The case of Turkish maritime industry. Expert Systems with Applications, 36, 190-198.

Celik, M., Kandakoglu, A., \& Er, D. (2009). Structuring fuzzy integrated multi-stages evaluation model on academic personnel recruitment in MET institutions. Expert Systems with Applications, 36, 6918-6927.

Che, Z., Wang, H., \& Chuang, C.-L. (2010). A fuzzy AHP and DEA approach for making bank loan decisions for small and medium enterprises in Taiwan. Expert Systems with Applications, 37, 7189-7199.

Chen, H., Lee, A., \& Tong, Y. (2007). Prioritization and operations NPD mix in a network with strategic partners under uncertainty. Expert Systems with Applications, 33, 337346.

Chen, L.-C., \& Chu, P.-Y. (2012). Developing the index for product design communication and evaluation from emotional perspectives. Expert Systems with Applications, 39, 2011-2020.

Chen, M.-K., \& Wang, S.-C. (2010). The use of a hybrid fuzzy-Delphi-AHP approach to develop global business intelligence for information service firms. Expert Systems with Applications, 37, 7394-7407.

Chia-Chi, S. (2010). A performance evaluation model by integrating fuzzy AHP and fuzzy TOPSIS methods. Expert Systems with Applications, 37, 7745-7754.

Chiang, T.-A., \& Che, Z. (2010). A fuzzy robust evaluation model for selecting and ranking NPD projects using Bayesian belief network and weight-restricted DEA. Expert Systems with Applications, 37, 7408-7418.

Cho, C., \& Lee, S. (2011). A study on process evaluation and selection model for business process management. Expert Systems with Applications, 38, 6339-6350.

Denton, L., \& Chan, A. (1991). Bank Selection Criteria of Multiple Bank Users in Hong Kong. International Journal of Bank Marketing, 9, 23 - 34.

Devlin, J., \& Gerrard, P. (2005). A study of customer choice criteria for multiple bank users. Journal of Retailing and Consumer Services, 12, 297-306. 
[Preprint version, please cite as] Alessio Ishizaka, Nam Nguyen Hoang, Calibrated Fuzzy AHP for current bank account selection, Expert Systems with Applications, DOI 10.1016/j.eswa.2012.12.089, advance online publication

Durán, O., \& Aguilo, J. (2008). Computer-aided machine-tool selection based on a FuzzyAHP approach. Expert Systems with Applications, 34, 1787-1794.

Duru, O., Bulut, E., \& Yoshida, S. Regime switching fuzzy AHP model for choice-varying priorities problem and expert consistency prioritization: A cubic fuzzy-priority matrix design. Expert Systems with Applications.

Farquhar, J., \& Panther, T. (2008). Acquiring and retaining customers in UK banks: An exploratory study. Journal of Retailing and Consumer Services, 15, 9-21.

Gupta, A., \& Dev, S. (2012). Client satisfaction in Indian banks: an empirical study. Management Research Review, 35, 617 - 636.

Hadi-Vencheh, A., \& Mohamadghasemi, A. (2011). A fuzzy AHP-DEA approach for multiple criteria ABC inventory classification. Expert Systems with Applications, 38, 3346-3352.

Haghighi, M., Divandari, A., \& Keimasi, M. (2010). The impact of 3D e-readiness on ebanking development in Iran: A fuzzy AHP analysis. Expert Systems with Applications, 37, 4084-4093.

Hinson, R., Owusu-Frimpong, N., \& Dasah, J. (2011). Brands and service-quality perception. Marketing Intelligence \& Planning, 29, 264 - 283.

Ho, C. (2012). Construct factor evaluation model of Health Management Center selected by customers with Fuzzy Analytic Hierarchy Process. Expert Systems with Applications, 39, 954-959.

Hosang, J. (2011). A fuzzy AHP-GP approach for integrated production-planning considering manufacturing partners. Expert Systems with Applications, 38, 5833-5840.

Hsu, S. H., Kao, C.-H., \& Wu, M.-C. (2009). Design facial appearance for roles in video games. Expert Systems with Applications, 36, 4929-4934.

Hsu, Y.-L., Lee, C.-H., \& Kreng, V. (2010). The application of Fuzzy Delphi Method and Fuzzy AHP in lubricant regenerative technology selection. Expert Systems with Applications, 37, 419-425.

Iç, Y. T., \& Yurdakul, M. (2009). Development of a decision support system for machining center selection. Expert Systems with Applications, 36, 3505-3513.

Isaai, M., Kanani, A., Tootoonchi, M., \& Afzali, H. (2011). Intelligent timetable evaluation using fuzzy AHP. Expert Systems with Applications, 38, 3718-3723.

Ishizaka, A., \& Labib, A. (2011). Review of the main developments in the analytic hierarchy process. Expert Systems with Applications, 38, 14336-14345.

Javanbarg, M., Scawthorn, C., Kiyono, J., \& Shahbodaghkhan, B. (2012). Fuzzy AHP-based multicriteria decision making systems using particle swarm optimization. Expert Systems with Applications, 39, 960-966.

Kamvysi, K., Gotzamani, K., Georgiou, A., \& Andronikidis, A. (2010). Integrating DEAHP and DEANP into the quality function deployment. The TQM Journal, 22, 293 - 316.

Katircioglu, S., Tumer, M., \& Kılınç, C. (2011). Bank selection criteria in the banking industry: an empirical investigation from customers in Romanian cities. African Journal of Business Management, 5, 5551-5558.

Katircioglu, S., Unlucan, F., \& Dalci, I. (2011). Bank selection factors in the banking industry : an empirical investigation from potential customers in Northern Cyprus. Acta oeconomica, 61, 77-89.

Kaya, T., \& Kahraman, C. (2011a). Fuzzy multiple criteria forestry decision making based on an integrated VIKOR and AHP approach. Expert Systems with Applications, 38, 7326-7333. 
[Preprint version, please cite as] Alessio Ishizaka, Nam Nguyen Hoang, Calibrated Fuzzy AHP for current bank account selection, Expert Systems with Applications, DOI 10.1016/j.eswa.2012.12.089, advance online publication

Kaya, T., \& Kahraman, C. (2011b). An integrated fuzzy AHP-ELECTRE methodology for environmental impact assessment. Expert Systems with Applications, 38, 8553-8562.

Kaya, T., \& Kahraman, C. (2011c). Multicriteria decision making in energy planning using a modified fuzzy TOPSIS methodology. Expert Systems with Applications, 38, 65776585.

Kennington, C., Hill, J., \& Rakowska, A. (1996). Consumer selection criteria for banks in Poland. International Journal of Bank Marketing, 14, 12-21.

Kilincci, O., \& Onal, S. (2011). Fuzzy AHP approach for supplier selection in a washing machine company. Expert Systems with Applications, 38, 9656-9664.

Kutlu, A., \& Ekmekçioğlu, M. (2012). Fuzzy failure modes and effects analysis by using fuzzy TOPSIS-based fuzzy AHP. Expert Systems with Applications, 39, 61-67.

Laroche, M., Rosenblatt, J., \& Manning, T. (1986). Services used and factors considered important in selecting a bank: an investigation across diverse demographic segments. International Journal of Bank Marketing, 4, 35-55.

Lee, A., Chen, W.-C., \& Chang, C.-J. (2008). A fuzzy AHP and BSC approach for evaluating performance of IT department in the manufacturing industry in Taiwan. Expert Systems with Applications, 34, 96-107.

Lee, J., \& Marlowe, J. (2003). How consumers choose a financial institution: decisionmaking criteria and heuristics. International Journal of Bank Marketing, 21, 53 - 71.

Lee, S.-H. (2010). Using fuzzy AHP to develop intellectual capital evaluation model for assessing their performance contribution in a university. Expert Systems with Applications, In Press, Corrected Proof, DOI: 10.1016/j.eswa.2009.1012.1020.

Lewis, B. (1981). Students' accounts: a profitable segment? European Journal of Marketing, 16, 63-72.

Li, S., \& Kuo, X. (2008). The inventory management system for automobile spare parts in a central warehouse. Expert Systems with Applications, 34, 1144-1153.

Li, T.-S., \& Huang, H.-H. (2009). Applying TRIZ and Fuzzy AHP to develop innovative design for automated manufacturing systems. Expert Systems with Applications, 36, 8302-8312.

Liu, C.-C., \& Chen, S.-Y. (2009). Prioritization of digital capital measures in recruiting website for the national armed forces. Expert Systems with Applications, 36, 94159421.

Lo, Y.-F., \& Wen, M.-H. (2010). A fuzzy-AHP-based technique for the decision of design feature selection in Massively Multiplayer Online Role-Playing Game development. Expert Systems with Applications, 37, 8685-8693.

Lu, W.-M., \& Wang, T.-C. (2011). A fuzzy multi-criteria model for the industrial cooperation program transaction strategies: A case in Taiwan. Expert Systems with Applications, $38,1490-1500$.

Lymperopoulos, C., Chaniotakis, I., \& Soureli, M. (2006). The importance of service quality in bank selection for mortgage loans. Managing Service Quality, 16, 365 - 379.

Mahmoud, M., Tweneboah-Koduah, E., \& Danku, C. (2011). Key motivations for bank loyaltys among university students in Ghana. Journal of Marketing Development and Competitiveness, 5, 96-107.

Manrai, L., \& Manrai, A. (2007). A field study of customers' switching behavior for bank services. Journal of Retailing and Consumer Services, 14, 208-215.

Mentes, A., \& Helvacioglu, I. (2012). Fuzzy decision support system for spread mooring system selection. Expert Systems with Applications, 39, 3283-3297. 
[Preprint version, please cite as] Alessio Ishizaka, Nam Nguyen Hoang, Calibrated Fuzzy AHP for current bank account selection, Expert Systems with Applications, DOI 10.1016/j.eswa.2012.12.089, advance online publication

Mokhlis, S., Salleh, H., \& Mat, N. (2011). What do young intellectuals look for in a bank? An empirical analysis of attribute importance in retail bank selection. Journal of Management Research, 3, 1-15.

Nepal, B., Yadav, O., \& Murat, A. (2010). A fuzzy-AHP approach to prioritization of CS attributes in target planning for automotive product development. Expert Systems with Applications, 37, 6775-6786.

Önüt, S., Efendigil, T., \& Soner Kara, S. (2010). A combined fuzzy MCDM approach for selecting shopping center site: An example from Istanbul, Turkey. Expert Systems with Applications, 37, 1973-1980.

Ou, J.-R., Fu, H.-P., Hu, K.-Y., Chu, K.-K., \& Chiou, C.-H. (2011). A study on performance tracing of the e-MP policy. Expert Systems with Applications, 38, 6606-6611.

Özkır, V., \& Demirel, T. (2012). A fuzzy assessment framework to select among transportation investment projects in Turkey. Expert Systems with Applications, 39, 74-80.

Paksoy, T., Pehlivan, N., \& Kahraman, C. (2012). Organizational strategy development in distribution channel management using fuzzy AHP and hierarchical fuzzy TOPSIS. Expert Systems with Applications, 39, 2822-2841.

Rostamzadeh, R., \& Sofian, S. (2011). Prioritizing effective 7Ms to improve production systems performance using fuzzy AHP and fuzzy TOPSIS (case study). Expert Systems with Applications, 38, 5166-5177.

Saaty, T. (1977). A scaling method for priorities in hierarchical structures. Journal of Mathematical Psychology, 15, 234-281.

Saaty, T. (1980). The Analytic Hierarchy Process. New York: McGraw-Hill.

Safakli, O. (2007). A research on the basic motivational factors in consumer bank selection: evidence from Northern Cyprus. Banks and Bank Systems, 2, 93-100.

Seçme, N., Bayrakdaroğlu, A., \& Kahraman, C. (2009). Fuzzy performance evaluation in Turkish Banking Sector using Analytic Hierarchy Process and TOPSIS. Expert Systems with Applications, 36, 11699-11709.

Şen, C., \& Çınar, G. (2010). Evaluation and pre-allocation of operators with multiple skills: A combined fuzzy AHP and max-min approach. Expert Systems with Applications, 37, 2043-2053.

Ta, H., \& Har, K. (2000). A study of bank selection decisions in Singapore using the Analytical Hierarchy Process. International Journal of Bank Marketing, 18, 170 - 180.

Thwaitesa, D., \& Verea, L. (1995). Bank selection criteria - a student perspective. Journal of Marketing Management, 11, 133-149.

Van Laarhoven, P., \& Pedrycz, W. (1983). A fuzzy extension of Saaty's priority theory. Fuzzy Sets and Systems, 11, 199-227.

Van Leekwijck, W., \& Kerre, E. (1999). Defuzzification: criteria and classification. Fuzzy Sets and Systems, 108, 159-178.

Wang, J., Fan, K., \& Wang, W. (2010). Integration of fuzzy AHP and FPP with TOPSIS methodology for aeroengine health assessment. Expert Systems with Applications, 37, $8516-8526$.

Wang, T.-C., \& Chen, Y.-H. (2011). Fuzzy multi-criteria selection among transportation companies with fuzzy linguistic preference relations. Expert Systems with Applications, 38, 11884-11890.

Wu, M.-C., Lo, Y.-F., \& Hsu, S.-H. (2008). A fuzzy CBR technique for generating product ideas. Expert Systems with Applications, 34, 530-540. 
[Preprint version, please cite as] Alessio Ishizaka, Nam Nguyen Hoang, Calibrated Fuzzy AHP for current bank account selection, Expert Systems with Applications, DOI 10.1016/j.eswa.2012.12.089, advance online publication

Yuen, K., \& Lau, H. (2011). A fuzzy group analytical hierarchy process approach for software quality assurance management: Fuzzy logarithmic least squares method. Expert Systems with Applications, 38, 10292-10302.

Zadeh, L. (1965). Fuzzy sets. Information and Control, 8, 338-353.

Zeydan, M., Çolpan, C., \& Çobanoğlu, C. (2011). A combined methodology for supplier selection and performance evaluation. Expert Systems with Applications, 38, 27412751. 